\title{
Ultrasound Elastography Using Three Images
}

\author{
Hassan Rivaz, Emad M. Boctor, Michael A. Choti, and Gregory D. Hager \\ Johns Hopkins University
}

\begin{abstract}
Displacement1 estimation is an essential step for ultrasound elastography and numerous techniques have been proposed to improve its quality using two frames of ultrasound RF data. This paper introduces a technique for calculating a displacement field from three frames of ultrasound RF data. To this end, we first introduce constraints on variations of the displacement field with time using mechanics of materials. These constraints are then used to generate a regularized cost function that incorporates amplitude similarity of three ultrasound images and displacement continuity. We optimize the cost function in an expectation maximization (EM) framework. Iteratively reweighted least squares (IRLS) is used to minimize the effect of outliers. We show that, compared to using two images, the new algorithm reduces the noise of the displacement estimation. The displacement field is used to generate strain images for quasi-static elastography. Phantom experiments and in-vivo patient trials of imaging liver tumors and monitoring thermal ablation therapy of liver cancer are presented for validation.
\end{abstract}

\section{Introduction}

Displacement, motion or time delay estimation in ultrasound images is an essential step in numerous medical imaging tasks including the rapidly growing field of imaging the mechanical properties of tissue [1]. In this work, we perform displacement estimation for quasi-static ultrasound elastography [1], which involves deforming the tissue slowly with an external mechanical force, imaging the tissue during the deformation, and performing displacement estimation using the images. More specifically, we focus on real-time freehand palpation elastography [2 7] where the external force is applied by simply pressing the ultrasound probe against the tissue. Ease of use, real-time performance and providing invaluable elasticity images for diagnosis and guidance/monitoring of surgical operations are the key factors that have led to its successful commercialization.

A typical ultrasound frame rate is $20-60 \mathrm{fps}$. As a result, an entire series of ultrasound images are freely available during the tissue deformation. Multiple ultrasound images have been used before to obtain strain images of highly compressed tissue by accumulating the intermediate strain images, and to obtain persistently high quality strain images by performing weighted averaging of the strain images 8 -10]. Accumulating and averaging strain images increases their signal to noise ratio (SNR) and contrast to noise ratio (CNR). However, these techniques are susceptible to drift, a problem with any sequential tracking system 

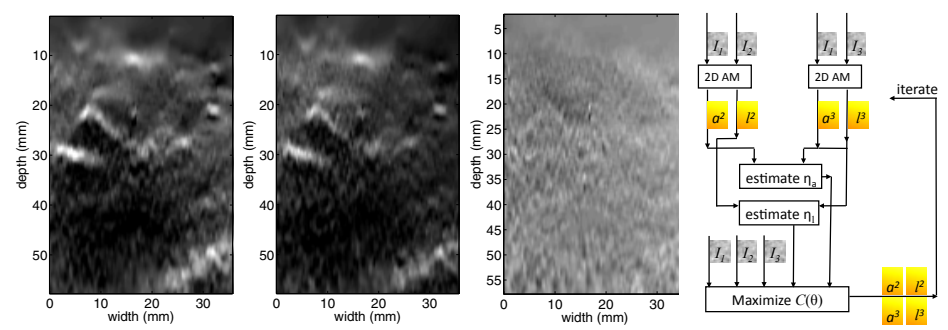

Fig. 1. Left: in-vivo images of liver. First and second ( $S_{1}$ and $S_{2}$ from left) are two strain fields calculated from $I_{1}$ and $I_{2}$, and from $I_{2}$ and $I_{3}$ respectively. $S_{1} \& S_{2}$ look "similar". Third image is $S_{1}-\eta S_{2}$ for $\eta=1.1$. The strain range in the first two images is 0 to $0.6 \%$, and in the third image is $\pm 0.3 \%$. Right shows the ElastMI algorithm.

[11]. In addition, these techniques do not exploit additional images to improve displacement estimation, which has many applications besides strain estimation. Time series of ultrasound data has also been used to characterize tissue 12] and improve elasticity reconstruction [13] and viscoelastic parameters [14, 15].

Figure 1 shows two consecutive strain images calculated from three ultrasound images using the 2D analytic minimization (AM) method [16]. Our motivation is to utilize the similarity of these two images to calculate a low variance displacement field from three images. The contributions of this work are: (1) introducing constraints on variation of the motion fields based on similarities of strain images through time; (2) proposing an EM algorithm to solve for motion fields using three images, and (3) reporting clinical tstudies of ablation guidance/monitoring, with data collection corresponding to before, during and after ablation.

The rest of this paper is summarized as follows. We first introduce the Elastography using Multiple Images (ElastMI) algorithm for tissue displacement estimation, which minimizes a cost function that incorporates data obtained from three images and exploits mechanical constraints. The estimated low variance displacement field can be used in numerous applications in imaging mechanical properties of tissue; we use it for generating strain images by calculating its spatial derivative. We use phantom and in-vivo clinical studies to compare ElastMI versus the recently developed elastography technique of 2D AM (code available online at www.cs.jhu.edu/ rivaz) [16].

\section{ElastMI: Elastography Using Multiple Images}

We have a set of $p=3$ images $I_{k}, k=1 \cdots 3$, each of size $m \times n$. Let the $2 \mathrm{D}$ displacement field $\mathbf{d}^{k}=\left(a^{k}, l^{k}\right)$ denote the displacement between $I_{k}$ and $I_{1}$, where $a$ refers to the axial (i.e. in the direction of the ultrasound beam) and $l$ to the lateral (i.e. perpendicular to the beam and in the imaging plane) directions. By the choice of reference $\mathbf{d}^{1}=0$. Note that we set $I_{1}$ as the reference image to simplify the notation. However, in our implementation we always take the middle image (i.e. $I_{2}$ ) as the reference. Our goal is to calculate a high quality $\mathbf{d}^{2}$ by utilizing all three images in a group-wise approach. 
It is well known that many tissue types display linear strain-stress relation in the 0 to $5 \%$ range (see [1] for example). In a freehand palpation elastography setup with ultrasound acquisition rate of $20 \mathrm{fps}$ or more, taking three consecutive images as $I_{1}, I_{2}, I_{3}$ corresponds to strain values of less than $1 \%$ and therefore the linearity assumption is valid. Using this property and some simplifying assumptions, it can be shown that the ratio of the strain and displacement fields in different times is a constant value, i.e. strain images are similar up to a scale as in Figure 1. We denote the scale factor by $\boldsymbol{\eta}=\left(\eta_{a}, \eta_{l}\right)$, and allow it to slightly change spatially to account for small nonlinearities in the tissue. As such, $\eta_{a}$ and $\eta_{l}$ are themselves scale fields in the axial and lateral directions each of size $m \times n$. Using this notation we have $a^{3}=\eta_{a} * a^{2}$ and $l^{3}=\eta_{l} \cdot * l^{2}$ where $*$ denotes point-wise multiplication.

Let $\boldsymbol{\theta}$ contain all the displacement unknowns $\mathbf{d}^{2}$ and $\mathbf{d}^{3}$. The MAP estimate of $\boldsymbol{\theta}$ is obtained by maximizing its posterior probability

$$
\operatorname{Pr}\left(\boldsymbol{\theta} \mid I_{1}, I_{2}, I_{3}\right) \propto \operatorname{Pr}\left(I_{1}, I_{2}, I_{3} \mid \boldsymbol{\theta}\right) \operatorname{Pr}(\boldsymbol{\theta})
$$

where we have ignored the normalization denominator. The data term is calculated as $\operatorname{Pr}\left(I_{1}, I_{2}, I_{3} \mid \boldsymbol{\theta}\right)=\Sigma_{\boldsymbol{\eta}} \operatorname{Pr}\left(I_{1}, I_{2}, I_{3}, \boldsymbol{\eta} \mid \boldsymbol{\theta}\right)$. The summation over the latent variable $\boldsymbol{\eta}$ makes the optimization problem intractable. We therefore use Expectation Maximization (EM) to make the problem tractable as following.

1. Initialize: find an estimate for $\boldsymbol{\theta}$ by applying the 2D AM method [16] to two pairs of images $\left(I_{1}, I_{2}\right)$ and $\left(I_{1}, I_{3}\right)$ independently.

2. E-step: find an estimate for $\boldsymbol{\eta}$ using $\boldsymbol{\theta}$ (details below).

3. M-step: update $\boldsymbol{\theta}$ with the current estimate of $\boldsymbol{\eta}$ (details below).

4. Iterate between 2 and 3 until convergence.

The algorithm is shown in Figure 1 right. Note that unlike the traditional EM which maximizes $\operatorname{Pr}\left(I_{1}, I_{2}, I_{3} \mid \boldsymbol{\theta}\right)$, we maximize the posterior probability of $\boldsymbol{\theta}$ (Equation 1). Steps 2 and 3 are elaborated below.

Calculating $\boldsymbol{\eta}$ from $\boldsymbol{\theta}$ Using Least Squares. At each sample $(i, j)$ in the displacement field $\mathbf{d}_{i, j}^{2}, i=1 \cdots m, j=1 \cdots n$ take a window of size $m_{w} \times n_{w}$ centered at the sample $\left(m_{w}\right.$ and $n_{w}$ are in the axial and lateral directions respectively and both are odd numbers). Stack the axial and lateral components of $\mathbf{d}_{i, j}^{2}$ that are in the window in two vectors $\mathbf{a}_{i, j}^{2}$ and $\mathbf{l}_{i, j}^{2}$, each of length $m_{w} \times n_{w}$. Similarly, generate $\mathbf{a}_{i, j}^{3}$ and $\mathbf{l}_{i, j}^{3}$ using $\mathbf{d}^{3}$. Note that since both displacement fields $\mathbf{d}_{i, j}^{2}$ and $\mathbf{d}_{i, j}^{3}$ are calculated with respect to samples on $I_{1}$, they correspond to the same sample $(i, j)$. We first calculate the axial component $\eta_{(i, j), a}$ $\left(\boldsymbol{\eta}_{(i, j)}=\left(\eta_{(i, j), a}, \eta_{(i, j), l}\right)\right)$. Discarding the spatial information in $\mathbf{a}_{i, j}^{2}$ and $\mathbf{a}_{i, j}^{3}$, we can average the two vectors into two scalers $\overline{\mathbf{a}}_{i, j}^{2}$ and $\overline{\mathbf{a}}_{i, j}^{3}$ and simply calculate $\eta_{(i, j), a}=\overline{\mathbf{a}}_{i, j}^{3} / \overline{\mathbf{a}}_{i, j}^{2}$. However, a more elegant way which also takes into account the spatial information is by calculating the least squares solution to the following over-determined problem (superscript $T$ denotes transpose).

$$
\mathbf{a}_{i, j}^{2} \eta_{(i, j), a}=\mathbf{a}_{i, j}^{3} \text { giving } \eta_{(i, j), a}=\frac{\mathbf{a}_{i, j}^{2 T} \mathbf{a}_{i, j}^{3}}{\mathbf{a}_{i, j}^{2 T} \mathbf{a}_{i, j}^{2}},
$$


which is what we use in our implementation. To calculate the ratio of the lateral displacement fields $\eta_{(i, j), l}$, we take into account possible lateral slip of the probe, which results in a rigid-body-motion. The rigid-body-motion can be simply calculated by averaging the lateral displacement in $\mathbf{d}_{i, j}^{2}$ and $\mathbf{d}_{i, j}^{3}$ in the entire image, and calculating the difference between these two average lateral displacements. The lateral scaling factor $\eta_{(i, j), l}$ can be calculated using an equation similar to 2 where the axial displacement $\mathbf{a}_{i, j}$ is replaced with the lateral displacements $\mathbf{l}_{i, j}$. However, we use the following approach which results in a better estimate for $\eta_{(i, j), l}$. The lateral strain $\epsilon_{l}$ is simply $\nu \epsilon_{a}$ where $\nu$ is an unknown Poisson's ratio. Since $\nu$ has a small dynamic range in soft tissue and since the difference between the two displacement maps $\mathbf{d}^{2}$ and $\mathbf{d}^{3}$ is small, we can assume that $\nu$ does not vary from $\mathbf{d}^{2}$ to $\mathbf{d}^{3}$. Therefore, $\eta_{(i, j), l}=\eta_{(i, j), a}$. This gives better estimate for $\eta_{(i, j), l}$ since axial displacement estimation is more accurate [16].

Calculating $\boldsymbol{\theta}$ by Maximizing Its Posterior Probability. To analytically solve the MAP estimate of $\boldsymbol{\theta}$, we assume that the data is independent and that the noise model is Gaussian. Although not completely held in real images, these assumptions are also the foundation behind sum of square difference and correlation based elastography methods, which have been extensively shown to produce reliable results. With these assumptions, the robust MAP estimate for $\boldsymbol{\theta}$ can be obtained by minimizing the following cost function

$$
\begin{aligned}
C(\boldsymbol{\theta})= & \sum_{i=1}^{m} w_{12, i}\left(I_{1}\left(\mathbf{x}_{i}\right)-I_{2}\left(\mathbf{x}_{i}+\hat{\mathbf{d}}_{i}^{2}\right)-\delta \mathbf{d}_{i}^{2 T} \nabla I_{2}\left(\mathbf{x}_{i}+\hat{\mathbf{d}}_{i}^{2}\right)\right)^{2}+ \\
& \sum_{i=1}^{m} w_{13, i}\left(I_{1}\left(\mathbf{x}_{i}\right)-I_{3}\left(\mathbf{x}_{i}+\eta_{i, a} \hat{\mathbf{d}}_{i}^{2}\right)-\eta_{i, a} \delta \mathbf{d}_{i}^{2 T} \nabla I_{3}\left(\mathbf{x}_{i}+\eta_{i, a} \hat{\mathbf{d}}_{i}^{2}\right)\right)^{2}+ \\
& \sum_{i=1}^{m}\left(\mathbf{d}_{i}^{2}-\mathbf{d}_{i-1}^{2}\right)^{T} A\left(\mathbf{d}_{i}^{2}-\mathbf{d}_{i-1}^{2}\right)
\end{aligned}
$$

where $\hat{\mathbf{d}}_{i}^{2}$ is the estimate obtained using $2 \mathrm{D} \mathrm{AM}, \delta \mathbf{d}_{i}^{2}=\mathbf{d}_{i}^{2}-\hat{\mathbf{d}}_{i}^{2}$ is the update in the displacement that we are looking for, $A=\operatorname{diag}(\alpha, \beta)$ is a $2 \times 2$ diagonal matrix with tunable regularization weights $(\alpha, \beta)$ that we adjust manually in this work, and $\nabla$ denotes the gradient operator. Robustness is achieved using IRLS through weights $w_{12, i}$ and $w_{13, i}$ which are calculated as following

$$
w_{1 k, i}=w\left(I_{1}\left(\mathbf{x}_{i}\right)-I_{k}\left(\mathbf{x}_{i}+\hat{\mathbf{d}}_{i}^{k}\right)\right) \text {, for } k=2,3, \text { and } w\left(r_{i}\right)=\left\{\begin{array}{cc}
1 & \left|r_{i}\right|<T \\
\frac{T}{\left|r_{i}\right|} & \left|r_{i}\right|>T
\end{array}\right.
$$

where $T$ is a tunable parameter which determines the residual level for which sample $i$ can be treated as outlier. A small $T$ will treat many samples as outliers.

Setting the derivative of $C$ w.r.t. the axial $\left(\delta a_{i}^{2}=\delta \mathbf{d}_{i, a}^{2}\right)$ and lateral $\left(\delta l_{i}^{2}=\right.$ $\left.\delta \mathbf{d}_{i, l}^{2}\right)$ components of $\delta \mathbf{d}_{i}^{2}$ for $i=1 \cdots m$ to zero and stacking the $2 m$ unknowns in $\delta \mathbf{d}^{2}=\left[\begin{array}{lllll}\delta a_{1}^{2} & \delta l_{1}^{2} \delta a_{2}^{2} \delta l_{2}^{2} \cdots \delta a_{m}^{2} & \delta l_{m}^{2}\end{array}\right]^{T}$ and the $2 m$ initial estimates in $\hat{\mathbf{d}}^{2}=$

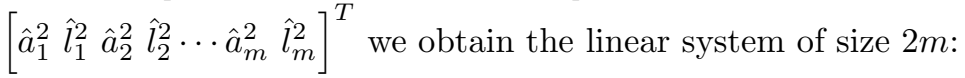


Table 1. The SNR and CNR of the strain images of Figure 2

\begin{tabular}{c|c|c|c|c} 
& Axial, 2D AM & Axial, ElastMI & Lateral, 2D AM & Lateral, ElastMI \\
\hline SNR & 11.11 & 12.64 & 6.06 & 6.63 \\
\hline CNR & 8.48 & 9.63 & 2.96 & 3.39
\end{tabular}

$$
\left(\mathscr{I}^{\prime}+\mathcal{D}\right) \delta \mathbf{d}^{2}=\boldsymbol{r}-\mathcal{D} \hat{\mathbf{d}}^{2}, \quad \mathcal{D}=\left[\begin{array}{cccccccc}
\alpha & 0 & -\alpha & 0 & 0 & 0 & \cdots & 0 \\
0 & \beta & 0 & -\beta & 0 & 0 & \cdots & 0 \\
-\alpha & 0 & 2 \alpha & 0 & -\alpha & 0 & \cdots & 0 \\
0 & -\beta & 0 & 2 \beta & 0 & -\beta & \cdots & 0 \\
0 & 0 & -\alpha & 0 & 2 \alpha & 0 & \cdots & 0 \\
\vdots & & & & & & \ddots & \\
0 & 0 & 0 & \cdots & -\alpha & 0 & \alpha & 0 \\
0 & 0 & 0 & \cdots & 0 & -\beta & 0 & \beta
\end{array}\right],
$$

where $\mathscr{I}^{\prime}$ is a symmetric tridiagonal matrix with $2 \times 2$ matrices $\mathfrak{I}^{\prime}$ in its diagonal:

$$
\begin{gathered}
\mathscr{I}^{\prime}=\operatorname{diag}\left(\mathfrak{I}^{\prime 2}(1) \cdots \mathfrak{I}^{\prime 2}(m)\right) \\
\mathfrak{I}^{\prime 2}(i)=\left[\begin{array}{cc}
\left(w_{12, i}+w_{13, i} \eta_{i, a}{ }^{2}\right) I_{1, a}^{\prime} & \left(w_{12, i}+w_{13, i} \eta_{i, a} \eta_{i, l}\right) I_{1, a}^{\prime} I_{1, l}^{\prime} \\
\left(w_{12, i}+w_{13, i} \eta_{i, a} \eta_{i, l}\right) I_{1, a}^{\prime} I_{1, l}^{\prime} & \left(w_{12, i}+w_{13, i} \eta_{i, l}{ }^{2}\right) I_{1, l}^{\prime}
\end{array}\right]
\end{gathered}
$$

where $I_{2}^{\prime}$ and $I_{3}^{\prime}$ are calculated respectively at $\left(\mathbf{x}_{i}+\hat{\mathbf{d}}_{i}^{2}\right)$ and at $\left(\mathbf{x}_{i}+\boldsymbol{\eta}_{i} * \hat{\mathbf{d}}_{i}^{2}\right)$, superscript $/$ indicates derivative and subscript $a$ and $l$ determine whether the derivation is in the axial or lateral direction, and $\boldsymbol{r}$ is a vector of length $2 m$ with elements:

$$
\begin{aligned}
i \text { even }: r_{i}= & w_{12, i} I_{1, a}^{\prime}\left(\mathbf{x}_{i}\right)\left[I_{1}\left(\mathbf{x}_{i}\right)-I_{2}\left(\mathbf{x}_{i}+\hat{\mathbf{d}}_{i}^{2}\right)\right]+ \\
& w_{13, i} \boldsymbol{\eta}_{i} * * I_{1, a}^{\prime}\left(\mathbf{x}_{i}\right)\left[I_{1}\left(\mathbf{x}_{i}\right)-I_{3}\left(\mathbf{x}_{i}+\boldsymbol{\eta}_{i} * \hat{\mathbf{d}}_{i}^{2}\right)\right] \\
i \text { odd }: r_{i}= & w_{12, i} I_{1, l}^{\prime}\left(\mathbf{x}_{i}\right)\left[I_{1}\left(\mathbf{x}_{i}\right)-I_{2}\left(\mathbf{x}_{i}+\hat{\mathbf{d}}_{i}^{2}\right)\right]+ \\
& w_{13, i} \boldsymbol{\eta}_{i} * I_{1, l}^{\prime}\left(\mathbf{x}_{i}\right)\left[I_{1}\left(\mathbf{x}_{i}\right)-I_{3}\left(\mathbf{x}_{i}+\boldsymbol{\eta}_{i} * \hat{\mathbf{d}}_{i}^{2}\right)\right] .
\end{aligned}
$$

The coefficient matrix in Equation 5 is pentadiagonal and symmetric. As such, it can be solved in $8 m$ operations, significantly less than $(2 m)^{3} / 3$ required for solving a full system. For all the results presented in this work, the EM algorithm is iterated once.

\section{Results of Phantom Experiments and Patient Trials}

RF data is acquired from an Antares Siemens system (Issaquah, WA) at the center frequency of $6.67 \mathrm{MHz}$ with a VF10-5 linear array at a sampling rate 


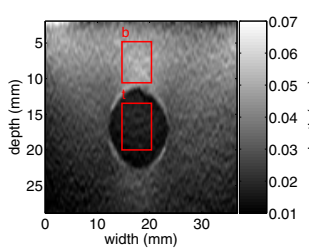

(a) 2D AM axial

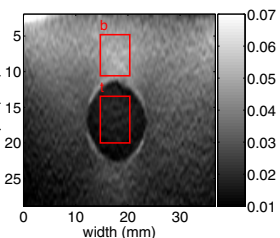

(b) ElastMI axial

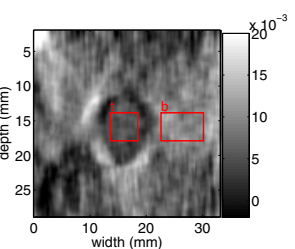

(c) 2D AM lateral

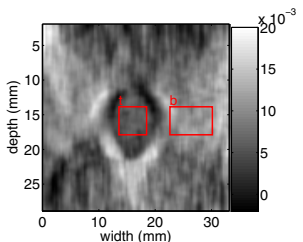

(d) ElastMI lateral

Fig. 2. Strain images of the CIRS phantom with the target and background windows (for calculation of SNR and CNR). No Kalman filter [16] is used to ease the comparison.

Table 2. The CNR of the strain images of first, second and third patient trials (images of second patient are shown in Figure 3). P1, P2 and P3 respectively correspond to patients 1,2 and 3. $2\left(\bar{s}_{b}-\bar{s}_{t}\right)^{2}$ and $\sigma_{b}^{2}+\sigma_{t}^{2}$ indicate contrast and noise respectively.

\begin{tabular}{c|c||c|c||c|c||c|c}
\multicolumn{2}{c}{} & before ablation & \multicolumn{2}{c||}{ during ablation } & \multicolumn{2}{c}{ after ablation } \\
\multicolumn{2}{c}{ 2D AM } & ElastMI & 2D AM & ElastMI & 2D AM & ElastMI \\
\hline P1 & $10^{4} \times 2\left(\bar{s}_{b}-\bar{s}_{t}\right)^{2}$ & - & - & - & - & 2.18 & 2.22 \\
& $10^{4} \times\left(\sigma_{b}^{2}+\sigma_{t}^{2}\right)$ & - & - & - & - & 0.108 & 0.083 \\
& $\mathrm{CNR}=\sqrt{\frac{2\left(\bar{s}_{b}-\bar{s}_{t}\right)^{2}}{\sigma_{b}^{2}+\sigma_{t}^{2}}}$ & - & - & - & - & 4.49 & 5.17 \\
\hline \multirow{2}{*}{$\mathrm{P} 2$} & $10^{4} \times 2\left(\bar{s}_{b}-\bar{s}_{t}\right)^{2}$ & 0.45 & 0.89 & - & - & 2.08 & 2.15 \\
& $10^{4} \times\left(\sigma_{b}^{2}+\sigma_{t}^{2}\right)$ & 0.0036 & 0.0045 & - & - & 0.204 & 0.142 \\
& $\mathrm{CNR}=\sqrt{\frac{2\left(\bar{s}_{b}-\bar{s}_{t}\right)^{2}}{\sigma_{b}^{2}+\sigma_{t}^{2}}}$ & 11.16 & 14.05 & - & - & 3.19 & 3.89 \\
\hline \multirow{2}{*}{$\mathrm{P} 3$} & $10^{4} \times 2\left(\bar{s}_{b}-\bar{s}_{t}\right)^{2}$ & 0.235 & 0.234 & 0.0745 & 0.1716 & 4.85 & 4.82 \\
& $10^{4} \times\left(\sigma_{b}^{2}+\sigma_{t}^{2}\right)$ & 0.0045 & 0.0036 & 0.0091 & 0.0161 & 0.204 & 0.171 \\
& $\mathrm{CNR}=\sqrt{\frac{2\left(\bar{s}_{b}-\bar{s}_{t}\right)^{2}}{\sigma_{b}^{2}+\sigma_{t}^{2}}}$ & 7.22 & 8.01 & 2.87 & 3.26 & 4.87 & 5.31
\end{tabular}

of $40 \mathrm{MHz}$. An elastography phantom (CIRS elastography phantom, Norfolk, VA) is compressed axially in two steps using a linear stage, and three images are acquired. Resulting strain images are shown in Figure 2, The unitless metric signal to noise ratio $\left(\mathrm{SNR}=\frac{\bar{s}_{b}}{\sigma_{b}}\right)$ and contrast to noise ratio $\left(\mathrm{CNR}=\sqrt{\frac{2\left(\bar{s}_{b}-\bar{s}_{t}\right)^{2}}{\sigma_{b}^{2}+\sigma_{t}^{2}}}\right)$ 1] of the ElastMI algorithm are shown in Table 1 (The SNR is only calculated for the background window). Comparing to the 2D AM, the ElastMI algorithm improves the SNR by approximately $14 \%$ and the CNR by approximately $11 \%$. The high quality of the lateral strain image, compared to state of the art strain imaging techniques, is visually noticeable.

In the clinical studies, RF data was acquired from ablation therapy of three patients with liver cancer using the Siemens Antares ultrasound machine in the following way: for the first patient only after ablation, for the second patient before and after ablation, and for the third patient before, during and after ablation. The ablation was administered using the RITA Model 1500 XRF generator (Rita Medical Systems, Fremont, CA). Tissue was simply compressed freehand at a frequency of approximately 1 compression per $2 \mathrm{sec}$ with the ultrasound probe without any attachment, and the strain images are generated offline. 

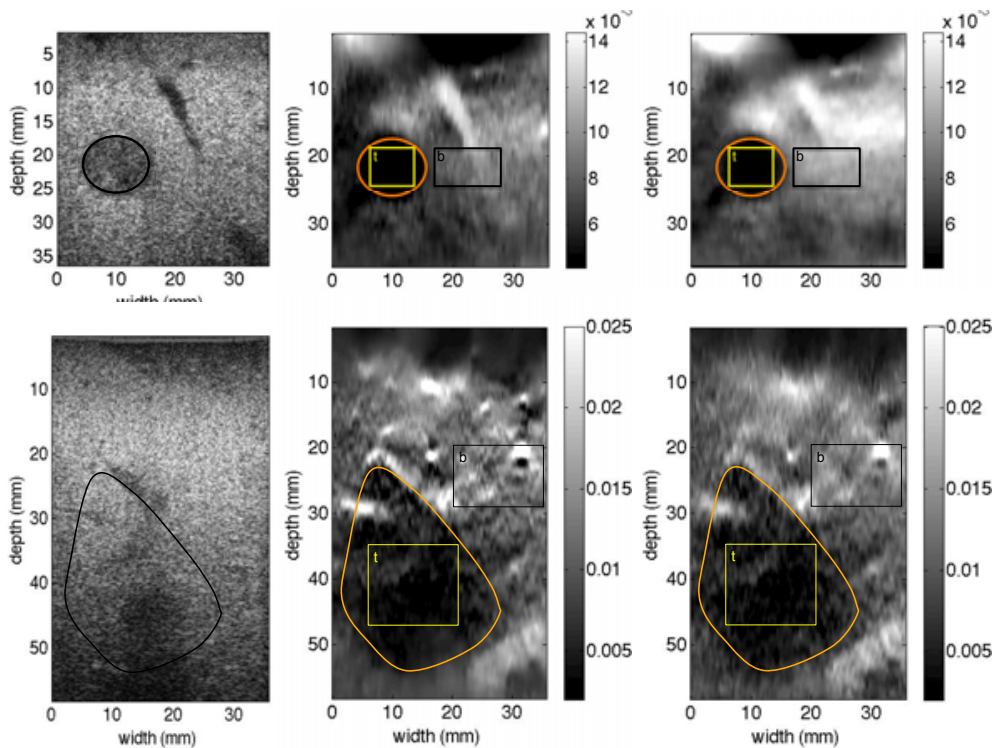

Fig. 3. Axial strain images of the second in-vivo patient trial corresponding to before (top row) and after (bottom row) ablation. The first, second and third columns are respectively B-mode, 2D AM strain and ElastMI strain images. The cancer tumor in the top row, and the ablated lesion in the bottom row are delineated. The CNR between the target and background (marked by t \& b) windows are given in Table 2.

Results of the second patient trial are shown in Figure 3 . Considering the numerous sources of noise in the clinical data, the high contrast of the tumor (top row) and the ablated lesion (bottom row) in the strain images make ElastMI a promising tool for both finding the tumor and monitoring the ablation. It should be noted that elastographic analysis of the ablated lesion is known to be challenging due to high temperatures which significantly degrade the quality of ultrasound data (mainly because of the air bubbles). Table 2 summarizes the CNR, as well as noise and contrast values, in the patient trials obtained using 2D AM and ElastMI methods. In the six cases presented in this table (two before ablation, one during ablation and three after ablation), the average increase in the CNR achieved using ElastMI compared to 2D AM is $17 \%$.

\section{Conclusions}

In this work, we proposed to utilize three ultrasound images to calculate high quality displacement fields. We neglected the dynamics of tissue motion and assumed a static model for tissue mechanics, which is valid in the quasi-static elastography. Using this model and assuming tissue linearity, which holds in the low strain rates of the freehand elastography, we introduced constraints on the variations of the strain field with time. We then proposed ElastMI, an EM algorithm that exploits these constraints for estimating displacement fields using 
three images. The algorithm involves solving sparse linear systems, and therefore runs in real-time. The low variance motion field that we compute by exploiting this new prior can be used in numerous applications in ultrasound imaging; we used it here to generate strain images.

Acknowledgements. H. Rivaz is supported by the Predoctoral Traineeship Award from the CDMRP and by the Advanced Simulation Fellowship from the Link Foundation. We thank P. Foroughi, I. Fleming and M. van Vledder for valuable discussions and anonymous reviewers for constructive feedback.

\section{References}

1. Greenleaf, J., Fatemi, M., Insana, M.: Selected methods for imaging elastic properties of biological tissues. Annu. Rev. Biomed. Eng. 5, 57-78 (2003)

2. Hall, T., Zhu, Y., Spalding, C.: In vivo real-time freehand palpation imaging. Ultrasound Med. Biol. 29, 427-435 (2003)

3. Zahiri, R., Salcudean, S.: Motion estimation in ultrasound images using time domain cross correlation with prior estimates. IEEE TBME 53, 1990-2000 (2006)

4. Rivaz, H., Boctor, E., Foroughi, P., Fichtinger, G., Hager, G.: Ultrasound elastography: a dynamic programming approach. IEEE Trans. Med. Imag. 27, 1373-1377 (2008)

5. Rivaz, H., Fleming, I., Assumpcao, L., Fichtinger, G., Hamper, U., Choti, M.A., Hager, G.D., Boctor, E.M.: Ablation monitoring with elastography: 2D in-vivo and 3D ex-vivo studies. In: Metaxas, D., Axel, L., Fichtinger, G., Székely, G. (eds.) MICCAI 2008, Part II. LNCS, vol. 5242, pp. 458-466. Springer, Heidelberg (2008)

6. Rivaz, H., Foroughi, P., Fleming, I., Zellars, R., Boctor, E., Hager, G.: Tracked regularized ultrasound elastography for targeting breast radiotherapy. In: Yang, G.-Z., Hawkes, D., Rueckert, D., Noble, A., Taylor, C. (eds.) MICCAI 2009. LNCS, vol. 5761, pp. 507-515. Springer, Heidelberg (2009)

7. Lindop, J., Treece, G., Gee, A., Prager, R.: 3D elastography using freehand ultrasound. Ultrasound Med. Biol. 32, 529-545 (2006)

8. Jiang, J., Hall, T., Sommer, A.: A novel image formation method for ultrasonic strain imaging. Ultrasound Med. Biol. 53, 1088-1102 (2007)

9. Lindop, J., Treece, G., Gee, A., Prager, R.: An intelligent interface for freehand strain imaging. Ultrasound Med. and Biol. 34, 1117-1128 (2008)

10. Chen, L., Housden, R., Treece, G., Gee, A., Prager, R.: A data weighting scheme for quasistatic ultrasound elasticity imaging. Univ. of Cambridge TR651 (2010)

11. Brown, M., Burschka, D., Hager, G.: Advances in computational stereo. IEEE Trans. Pattern Anal. Mach. Intell. 25, 993-1008 (2003)

12. Moradi, M., Mousavi, P., Abolmaesui, P.: Tissue typing using ultrasound rf time series: Experiments with animal tissue samples. Med. Phys. 37, 1401-1413 (2010)

13. Li, L., Cui, Y., Kadour, M., Noble, A.: Elasticity reconstruction from displacement and confidence measures of a multi-compressed ultrasound rf sequence. IEEE Trans. Ultrason. Ferroelectr. Freq. Control 55, 319-326 (2008)

14. Catheline, S., et al.: Measurement of viscoelastic properties of homogeneous soft solid using transient elastography. J. Acoust. Soc. Amer. 116, 3734-3741 (2004)

15. Eskandari, H., Salcudean, S., Rohling, R.: Viscoelastic parameter estimation based on spectral analysis. IEEE T. Ultrason. Ferro. Freq. Control 55, 1611-1625 (2008)

16. Rivaz, H., Boctor, E.M., Choti, M.A., Hager, G.D.: Real-time regularized ultrasound elastography. IEEE Trans. Med. Imaging 30, 928-945 (2011) 